\title{
Satellite Mapping of Urban Expansion and Landscape Changes in Beijing, China: A Longitudinal Study
}

\author{
Xiaojun Yang ${ }^{\mathrm{a}}$ *, Ting Liu ${ }^{\mathrm{b}}$ \\ ${ }^{a}$ Department of Geography, Florida State University, USA; xyang@fsu.edu \\ ${ }^{b}$ Department of Geography and Environmental Studies, Northeastern Illinois University, USA; t-liul@neiu.edu \\ * Corresponding author
}

Keywords: Urban expansion, satellite mapping, Beijing, China, environmental sustainability

\begin{abstract}
:
Urbanization has been recognized as a major form of global environmental changes. With the concentration of large population in urban areas, there have been increasing concerns over the negative impacts of urbanization that collectively degrade the quality of our living environment. Over the past two decades, urban expansion has been a subject in numerous studies mostly through using remote sensing technology. Although many cities, large or small, have been targeted, Beijing as China's capital city has probably been more frequently researched than any other metropolises in the world. This may be due to a myriad of ecological and societal pressures accompanied with the rapid urban expansion in the Beijing metropolitan area since late 1970s when China initiated the open-door policy and economic transformation, which attracted attentions from both the academic community and the society. This paper aims to examine some major advances in remote sensing-based urban expansion studies with Beijing as the focus. For this purpose, we surveyed peer-reviewed English literature paying attention on some journal articles reporting the subject. Specifically, we examined the progress on several issues related to the research design and implementation, i.e., spatial extent or temporal scale, data sources, and quantified dimensions. Based on the literature review, we further identified several major challenges and discussed some future research directions. It is believed that such a longitudinal study focusing on major English literature examining the urbanization pattern in Beijing through remote sensing can not only help accomplish better research design in the subject but also assist formulating effective strategies and polices to deal with major challenges towards environmental sustainability in large metropolises across the world.
\end{abstract}

